\title{
A faster FPT algorithm for Bipartite Contraction $^{\star}$
}

\author{
Sylvain Guillemot and Dániel Marx \\ Institute for Computer Science and Control, Hungarian Academy of Sciences \\ (MTA SZTAKI), Budapest, Hungary
}

\begin{abstract}
The Bipartite Contraction problem is to decide, given a graph $G$ and a parameter $k$, whether we can can obtain a bipartite graph from $G$ by at most $k$ edge contractions. The fixed-parameter tractability of the problem was shown by Heggernes et al. 13, with an algorithm whose running time has double-exponential dependence on $k$. We present a new randomized FPT algorithm for the problem, which is both conceptually simpler and achieves an improved $2^{O\left(k^{2}\right)} n m$ running time, i.e., avoiding the double-exponential dependence on $k$. The algorithm can be derandomized using standard techniques.
\end{abstract}

\section{Introduction}

A graph modification problem aims at transforming an input graph into a graph satisfying a certain property, by at most $k$ operations. These problems are typically studied from the viewpoint of fixed-parameter tractability, where the goal is to obtain an algorithm with running time $f(k) n^{c}$ (or FPT algorithm). Here, $f(k)$ is a computable function depending only on the parameter $k$, which confines the combinatorial explosion that is seemingly inevitable for an NP-hard problem. The most intensively studied graph modification problems involve vertexor edge-deletions as their base operation; fixed-parameter tractability has been established for the problems of transforming a graph into a forest [11]8] , a bipartite graph [28/11/21/1720/27/15, a chordal graph 23/16, a planar graph [25|14], a unit/proper interval graph [29|30, or an interval graph [31|2]. Results have also been obtained for problems involving directed graphs [5] or grouplabeled graphs [1017.

Recently, there has been an interest in graph modification problems involving edge contractions. These problems fall in the following general framework. Given a graph property $\Pi$, the problem $\Pi$-CONTRACTION is to decide, for a graph $G$ and a parameter $k$, whether we can obtain a graph in $\Pi$, by starting from $G$ and performing at most $k$ edge contractions. For each graph property $\Pi$ admitting a polynomial recognition algorithm, it is then natural to ask whether $\Pi$-CONTRACTION admits an FPT algorithm. Such algorithms have been given

\footnotetext{
* Research supported in part by the European Research Council (ERC) grant "PARAMTIGHT: Parameterized complexity and the search for tight complexity results," reference 280152 and OTKA grant NK105645.
} 
when $\Pi$ is the class of paths, the class of trees [12], the class of planar graphs 9], or the class of bipartite graphs [13].

For the case of bipartite graphs, the problem is called BiparTite ConTraCTION, and Heggernes et al. 13] obtained an FPT algorithm with a running time double-exponential in $k$. The algorithm combines several tools from parameterized algorithmics, such as the irrelevant vertex technique, important separators, treewidth reduction, and Courcelle's theorem. In this note, we present a new FPT algorithm for the problem, which is both conceptually simpler and faster. Similar to the compression routine for OdD CyCle Transversal in [28, we reduce Bipartite Contraction to several instances of an auxiliary cut problem. Our main effort is spent on obtaining an FPT algorithm for this cut problem. This is achieved by using the notion of important separators from [22, together with the randomized coloring technique introduced by Alon et al [1]. We obtain the following result:

Theorem 1. BIPARTITE CONTRACTION has a randomized FPT algorithm with running time $2^{O\left(k^{2}\right)} n m$ and a deterministic algorithm with running time $2^{O\left(k^{2}\right)} n^{O(1)}$.

This paper is organized as follows. We first introduce the relevant notation and definitions in Section 2, We explain in Section 3 how Bipartite ConTRACTION can be reduced to several instances of a suitable cut problem called RANK-Cut. In Section 4, we define a constrained version of the RANK-Cut problem and show that it is polynomial-time solvable. In Section [5 we present a randomized reduction of RANK-CUT to its constrained version. Finally, in Section 6 we derandomize this reduction and we complete the proof of Theorem 1. Section 7 concludes the paper.

\section{Preliminaries}

Given a graph $G$, we let $V(G)$ and $E(G)$ denote its vertex set and edge set, respectively. We let $n=|V(G)|$ and $m=|E(G)|$. Given $X \subseteq V(G)$, we denote by $G[X]$ the subgraph of $G$ induced by $X$, and we denote by $G \backslash X$ the subgraph of $G$ induced by $V(G) \backslash X$. Given a set $F \subseteq E(G)$ of edges, we denote by $V(F)$ the endpoints of the edges in $F$, and we say that $F$ spans the vertices in $V(F)$. Given $F \subseteq E(G)$, we denote by $G[F]$ the graph with vertex set $V(F)$ and edge set $F$; we denote by $G \backslash F$ the graph with vertex set $V(G)$ and edge set $E(G) \backslash F$. For an edge $e$, we denote by $G / e$ the graph obtained by contracting edge $e$, that is, by removing the endpoints $u$ and $v$ of $e$ and introducing a new vertex that is adjacent to every vertex that is adjacent to at least one of $u$ or $v$. Given $F \subseteq E(G)$, we denote by $G / F$ the graph obtained from $G$ by contracting all the edges of $F$; it is easy to observe that the graph $G / F$ does not depend on the order in which we perform the contractions.

Fix two disjoint subsets of vertices $X, Y$ of a graph $G$. An $(X, Y)$-walk in $G$ is a sequence $W=v_{0} v_{1} \ldots v_{\ell}$ of vertices such that $v_{0} \in X, v_{\ell} \in Y$, and $v_{i} v_{i+1} \in E(G)$ for $0 \leq i<\ell$; the length of $W$ is $\ell$, and we call $W$ an $(X, Y)$ path in $G$ if the vertices $v_{i}$ are pairwise distinct. We will simply use the term 
"path" when the sets $X, Y$ are irrelevant. An $(X, Y)$-cut in $G$ is defined as a set $C \subseteq E(G)$ such that $G \backslash C$ has no $(X, Y)$-path; an $(X, Y)$-separator in $G$ is defined as a set $S \subseteq V(G) \backslash(X \cup Y)$ such that $G \backslash S$ has no $(X, Y)$-path. Note that the $(X, Y)$-separator is by definition disjoint from $X$ and $Y$. An $(X, Y)$-cut (resp. $(X, Y)$-separator) $C$ is inclusion-wise minimal if no proper subset of $C$ is an $(X, Y)$-cut (resp. $(X, Y)$-separator).

A bipartite modulator of $G$ is a set $F \subseteq E(G)$ such that $G \backslash F$ is bipartite. The rank of a graph $G$ is the number of edges of a spanning forest of $G$. The rank of a set $F \subseteq E(G)$ of edges, denoted by $r(F)$ is the rank of $G[F]$. As observed in 24, we can alternatively define BipARTite Contraction as the following problem: given a graph $G$ and an integer $k$, find a bipartite modulator $F$ of $G$ such that $r(F) \leq k$. We reproduce the proof here for completeness.

Lemma 2. The following statements are equivalent:

(i) there exists a set $F \subseteq E(G)$ such that $|F| \leq k$ and $G / F$ is bipartite;

(ii) there exists a set $F \subseteq E(G)$ such that $r(F) \leq k$ and $G \backslash F$ is bipartite.

Proof. $(i) \Rightarrow(i i)$ : Let $F^{\prime}$ denote the edges of $G$ having both endpoints in a same connected component of $G[F]$. Observe that $r\left(F^{\prime}\right) \leq k$, as $F$ contains a spanning forest of $G\left[F^{\prime}\right]$. We claim that $G \backslash F^{\prime}$ is bipartite. Observe that the vertex set of each connected component of $G[F]$ is an independent set in $G \backslash F^{\prime}$. Therefore, a proper 2-coloring of $G / F$ can be turned into a proper 2-coloring of $G \backslash F^{\prime}$ if we color every vertex in a connected component $K$ of $G[F]$ by the color of the single vertex corresponding to $K$ in $G / F$.

$($ ii $) \Rightarrow(i)$ : Let us fix a proper 2 -coloring of $G \backslash F$. We can assume that $F$ is a minimal set of edges such that $G \backslash F$ is bipartite. Therefore, in each connected component of $G[F]$, every vertex has the same color in the 2-coloring of $G \backslash F$. Hence, contracting each connected component of $F$ to a single vertex gives a bipartite graph. This graph can be obtained by contracting the edges of a spanning forest of $F$, which has $r(F) \leq k$ edges.

One can deduce from Lemma 2 that BipARTite Contraction is a monotone problem in the sense that positive instances are preserved by taking (not necessarily induced) subgraphs. Such a monotonicity property is typically required when the problem is solved using the iterative compression technique (see, e.g., [1113]). As we shall see in Section 3, even though we solve the problem by "compressing" from a set of vertices $X$ whose deletion makes the graph bipartite, we do not use iterative compression as we perform a single compression step from a set $X$ obtained with a black-box algorithm. Hence, we do not explicitly need the monotonicity property in this paper.

\section{Reduction to a cut problem}

We first define a compression version of the problem, named BIPARTITE CoNTRACTION COMPRESSION: given a graph $G$, an integer $k$, and a set $X \subseteq V(G)$ 
with $|X| \leq 2 k$ such that $G \backslash X$ is bipartite, is there a bipartite modulator $F$ of $G$ with $r(F) \leq k$ ? The following lemma establishes how a compression routine for the problem entails the fixed-parameter tractability of BIPARTITE CONTRACTION.

Lemma 3. Suppose that Bipartite Contraction Compression is solvable in time $T(k, n, m)$. Then BipaRTite ContraCtion is solvable in time $O\left(9^{k} k n m+\right.$ $T(k, n, m))$.

Proof. An instance $I=(G, k)$ of Bipartite Compression is solved the following way. First, we run the algorithm of Reed et al. 28] to look for a set $X \subseteq V(G)$ of size $\leq 2 k$ such that $G \backslash X$ is bipartite; the running time of the algorithm is $O\left(3^{2 k} \mathrm{knm}\right)$ (see also [21] $)^{1}$. If there is no such set, then we answer "no". Otherwise, we run the algorithm for Bipartite Contraction Compression on $(G, k, X)$. This takes time $O\left(9^{k} k n m+T(k, n, m)\right)$ as claimed. The correctness of this algorithm follows by observing that, if $F$ is a solution for instance $I$ of Bipartite Contraction, then $X=V(F)$ is a set of size at most $2 k$ such that $G \backslash X$ is bipartite.

In the rest of this section, we concentrate on the Bipartite Contraction COMPRESSION problem. We solve the problem similarly to the compression routine for Odd Cycle Transversal [28]. First, we adapt the construction of 28] to the case of edge sets.

Suppose that we are given a graph $G$ in which a bipartite modulator has to be found, along with a set $X \subseteq V(G)$ such that $G \backslash X$ is bipartite. We construct a graph $G^{\prime}$ as follows. Let $S_{1}, S_{2}$ be a bipartition of $G \backslash X$, and let $<$ be an arbitrary total ordering of $V(G)$. We let $V\left(G^{\prime}\right)=(V(G) \backslash X) \cup X^{\prime}$ with $X^{\prime}=\left\{x_{1}, x_{2}: x \in X\right\}$, and $E\left(G^{\prime}\right)=E(G \backslash X) \cup\left\{u v_{3-i}: u v \in E, u \in\right.$ $\left.S_{i}, v \in X\right\} \cup\left\{u_{1} v_{2}: u v \in E, u, v \in X, u<v\right\}$. Observe that $G^{\prime}$ is bipartite, with bipartition $S_{1}^{\prime}=S_{1} \cup\left\{x_{1}: x \in X\right\}$ and $S_{2}^{\prime}=S_{2} \cup\left\{x_{2}: x \in X\right\}$. Furthermore, if we identify $x_{1}$ with $x_{2}$ for every $x \in X$ in $G^{\prime}$, then we get the graph $G$; in particular, $G$ and $G^{\prime}$ have the same number of edges.

Define a bijection $\Phi: E(G) \rightarrow E\left(G^{\prime}\right)$ which preserves each edge of $E(G \backslash X)$, maps each edge $u v \in E(G)\left(u \in S_{i}, v \in X\right)$ to the edge $u v_{3-i}$, and maps each edge $u v \in E(G)(u, v \in X, u<v)$ to the edge $u_{1} v_{2}$. We say that a partition of $X^{\prime}$ into two sets $X_{A}^{\prime}, X_{B}^{\prime}$ is valid if for each $x \in X$, exactly one of $\left\{x_{1}, x_{2}\right\}$ is in $X_{A}^{\prime}$. The following lemma is similar to Lemma 1 of [28].

Lemma 4. For every $F \subseteq E(G)$, the following statements are equivalent:

(i) $G \backslash F$ is bipartite,

\footnotetext{
${ }^{1}$ Very recently, two linear-time algorithms for Odd CyCle Transversal were announced 27/15. Also, the dependence on $k$ was improved from $3^{k}$ to $2.3146^{k}$ 20. However, using any of these algorithms here would not improve the overall asymptotic running time of our algorithm, as the dominating term comes from the RANKCuT algorithm of Theorem 12
} 
(ii) There is a valid partition $X_{A}^{\prime}, X_{B}^{\prime}$ of $X^{\prime}$ such that $\Phi(F)$ is an $\left(X_{A}^{\prime}, X_{B}^{\prime}\right)$-cut in $G^{\prime}$.

Proof. $(i) \Rightarrow(i i)$. Suppose that $G \backslash F$ is bipartite with bipartition $V_{1}, V_{2}$. Define the partition $X_{A}^{\prime}, X_{B}^{\prime}$ of $X^{\prime}$ such that: for $u \in X, u_{1} \in X_{A}^{\prime}$ iff $u \in V_{1}$. Observe that $X_{A}^{\prime}, X_{B}^{\prime}$ is a valid partition of $X^{\prime}$. We claim that $C=\Phi(F)$ is an $\left(X_{A}^{\prime}, X_{B}^{\prime}\right)$ cut in $G^{\prime}$. Towards a contradiction, assume that $G^{\prime} \backslash C$ contains an $X_{A}^{\prime}, X_{B}^{\prime}$-path $P^{\prime}$. Suppose that the endpoints of $P^{\prime}$ are $u_{i} \in X_{A}^{\prime}, v_{j} \in X_{B}^{\prime}$; then $u \in V_{i}, v \in$ $V_{3-j}$. The path $P^{\prime}$ corresponds to an $u, v$-path $P$ in $G \backslash F$, of the same length. If $j=i$, then $u_{i}, v_{j}$ both belong to $S_{i}^{\prime}$, and we obtain that $P$ is a path of even length between $V_{i}$ and $V_{3-i}$, contradiction. If $j=3-i$, then $u_{i} \in S_{i}^{\prime}, v_{j} \in S_{3-i}^{\prime}$, and we obtain that $P$ is a path of odd length between $V_{i}$ and $V_{i}$, contradiction. We conclude that $C$ is an $\left(X_{A}^{\prime}, X_{B}^{\prime}\right)$-cut in $G^{\prime}$, as claimed.

(ii) $\Rightarrow(i)$. Suppose that $C \subseteq E\left(G^{\prime}\right)$ is an $\left(X_{A}^{\prime}, X_{B}^{\prime}\right)$-cut in $G^{\prime}$, for some valid partition $X_{A}^{\prime}, X_{B}^{\prime}$ of $X^{\prime}$. We claim that $F=\Phi^{-1}(C)$ is such that $G \backslash F$ is bipartite. We define a 2-coloring $\chi$ of $G \backslash F$ as follows: (1) If $u \in X$, then $\chi(u)=1$ iff $u_{1} \in X_{A}^{\prime}$; (2) If $u \in V \backslash X$ is reachable from $X_{A}^{\prime}$ in $G^{\prime} \backslash C$, then $\chi(u)=1$ iff $u \in S_{1}$; (3) If $u \in V \backslash X$ is not reachable from $X_{A}^{\prime}$ in $G^{\prime} \backslash C$, then $\chi(u)=1$ iff $u \in S_{2}$. We verify that $\chi$ is a proper 2-coloring of $G \backslash F$. Consider an edge $u v \in E(G) \backslash F$, there are three cases. If $u, v \notin X$, then $u \in S_{i}, v \in S_{3-i}$; as $u, v$ are either both in case (2) or both in case (3), it follows that $\chi(u) \neq \chi(v)$. If $u \in S_{i}, v \in X$, then $u v_{3-i}$ is an edge of $G^{\prime} \backslash C$; if $v_{3-i} \in X_{A}^{\prime}$ then $\chi(v)=3-i$ by (1), and $\chi(u)=i$ by (2); if $v_{3-i} \in X_{B}^{\prime}$ then $\chi(v)=i$ by $(1)$, and $\chi(u)=3-i$ by (3). If $u, v \in X$ with $u<v$, then $u_{1} v_{2}$ is an edge of $G^{\prime} \backslash C$, and thus we have $u_{1}, v_{2}$ both in $X_{A}^{\prime}$ or both in $X_{B}^{\prime}$, which implies that $\chi(u) \neq \chi(v)$. We conclude that $G \backslash F$ is bipartite, as claimed.

Lemma 4 turns the problem of finding a bipartite modulator into several instances of a cut problem (one for each valid partition). The same way as Lemma 2 shows the equivalence of Bipartite CONTRACTION with the problem of finding a bipartite modulator with a rank constraint, we show that Lemma 4 allows us to solve Bipartite Contraction Compression by solving a cut problem with a rank constraint. However, there is a technical detail related to the fact that two vertices $x_{1}, x_{2} \in X^{\prime}$ correspond to each vertex $x \in X$ in the construction of $G^{\prime}$; we need the following definition to deal with this issue. Let $M, F \subseteq E(G)$ be two subsets of edges. We define the $M$-rank $r_{M}(F)$ of $F$ as the rank of the graph $G[F \cup M] / M$. Our auxiliary problem is defined as follows.

RANK-CuT
Input: A graph $G$, an integer $k$, two sets $X, Y \subseteq V(G)$, and a set
\[ M \subseteq E(G) \text { with }|M| \leq 2 k \]
Question: Is there an $(X, Y)$-cut $C$ in $G$ such that $r_{M}(C) \leq k ?$

The following simple observation will be useful later:

Lemma 5. If $|M| \leq 2 k$ and $r_{M}(C) \leq k$, then $C \cup M$ spans at most $6 k$ vertices. 
Proof. Each contraction can decrease rank by at most one, hence the rank of $G[C \cup M]$ is at most $3 k$. As $G[C \cup M]$ has no isolated vertices by definition, it follows that $G[C \cup M]$ has at most $6 k$ vertices.

We now describe how an FPT algorithm for RANK-CUT yields an FPT algorithm for Bipartite Contraction Compression; Section 46 6how the fixed-parameter tractability of RANK-CUT itself.

Lemma 6. Suppose that RANK-CUT is solvable in time $T(k, n, m)$. Then BIPARTite Contraction Compression is solvable in $O\left(4^{k}(T(k, n, m)+n+m)\right)$ time.

Proof. Consider an instance $I=(G, k, X)$ of Bipartite Contraction ComPRESSION. From $G$ and $X$, we construct the graph $G^{\prime}$ as described above Lemma 4 . We let $H$ be obtained from $G^{\prime}$ by adding the edge $x_{1} x_{2}$ for every $x \in X$; let $M \subseteq E(H)$ be the set of these new edges.

We solve Bipartite Contraction Compression by the following algorithm. For each valid partition $X_{A}^{\prime}, X_{B}^{\prime}$ of $X^{\prime}$, we run the algorithm for RANKCut on the instance $I^{\prime}=\left(H, k, X_{A}^{\prime}, X_{B}^{\prime}, M\right)$. We answer "yes" if and only if one of the instances $I^{\prime}$ was a yes-instance of RANK-CUT. Note that, as $|X| \leq 2 k$ by assumption, we have $|M| \leq 2 k$ and thus each instance $I^{\prime}$ is a valid instance of RANK-Cut. As there are $2^{|X|} \leq 4^{k}$ valid partitions of $X^{\prime}$, the claimed running time follows. We show that it correctly solves BipARTite Contraction Compression.

Suppose that $I$ admits a solution $F$ with $r(F) \leq k$, then $G \backslash F$ is bipartite. Thus, by Lemma 4 there exists a valid partition $X_{A}^{\prime}, X_{B}^{\prime}$ of $X^{\prime}$ such that $\Phi(F)$ is an $\left(X_{A}^{\prime}, X_{B}^{\prime}\right)$-cut in $G^{\prime}$. Hence, $C=\Phi(F) \cup M$ is an $\left(X_{A}^{\prime}, X_{B}^{\prime}\right)$-cut in $H$, and since $H[C] / M$ is isomorphic to $G[F]$, we have $r_{M}(C)=r(F) \leq k$. It follows that $C$ is a solution for RANK-CUT on the instance $I^{\prime}=\left(H, X_{A}^{\prime}, X_{B}^{\prime}, M, k\right)$. Conversely, suppose that $C$ is a solution of RANK-CUT on the instance $I^{\prime}=$ $\left(H, X_{A}^{\prime}, X_{B}^{\prime}, M, k\right)$, for some valid partition $X_{A}^{\prime}, X_{B}^{\prime}$ of $X^{\prime}$. Observe that $M \subseteq C$ (as each edge of $M$ is between $X_{A}^{\prime}$ and $X_{B}^{\prime}$ ), and that $C \backslash M$ is an $\left(X_{A}^{\prime}, X_{B}^{\prime}\right)$-cut in $H \backslash M=G^{\prime}$. Thus, if we define $F=\Phi^{-1}(C \backslash M)$, we obtain that $G \backslash F$ is bipartite by Lemma 4. Observe that contracting the edges of $M$ in $H[C]$ gives a graph isomorphic to $G[F]$. Therefore, $r(F)=r_{M}(C) \leq k$, and we conclude that $F$ is a solution for the instance $I$.

\section{Solving a constrained version of Rank-Cut}

In this section, we introduce a constrained variant of RANK-CUT, and show its polynomial-time solvability. We give a randomized reduction of RANK-CUT to this variant in the next section. In the constrained problem, the cut has to be constructed as the union of disjoint components prescribed in the input: 
CONSTRAined RANK-CuT

Input: A graph $G$, an integer $k$, two subsets $X, Y \subseteq V(G)$, a set $M \subseteq E(G)$, and a partition $P=\left(V_{1}, \ldots, V_{\ell}\right)$ of $V(G)$ such that

(i) $G\left[V_{i}\right]$ is connected for every $1 \leq i \leq \ell$, and

(ii) there is no edge of $M$ between $V_{i}$ and $V_{j}$ for any $i \neq j$.

Question: Is there a set $Z \subseteq\{1, \ldots, \ell\}$ such that $C_{Z}=\cup_{i \in Z} E\left(G\left[V_{i}\right]\right)$ is an $(X, Y)$-cut in $G$ with $r_{M}\left(C_{Z}\right) \leq k$ ?

Note that a $V_{i}$ can consist of a single vertex, in which case $E\left(G\left[V_{i}\right]\right)=\emptyset$ and it does not matter if $i$ is in $Z$ or not. We show that the constrained problem can be reformulated as a weighted separator problem between two sets, and hence can we solved in polynomial time.

Lemma 7. Constrained RANK-Cut can be solved in $O(k(n+m))$ time.

Proof. Let $I=(G, k, X, Y, M, P)$ be an instance of Constrained Rank-Cut with $P=\left(V_{1}, \ldots, V_{\ell}\right)$. Starting with $G$, we build a weighted graph $G^{\prime}$ as follows:

- we remove the edges of $\cup_{i=1}^{\ell} E\left(G\left[V_{i}\right]\right)$;

- we give an infinite weight to the vertices of $V(G)$;

- for each $1 \leq i \leq \ell$, we add a vertex $v_{i}$ of weight $r_{M}\left(E\left(G\left[V_{i}\right]\right)\right)$, and we make $v_{i}$ adjacent to the vertices of $V_{i}$.

We answer "yes" if and only if $G^{\prime}$ has an $(X, Y)$-separator of weight at most $k$. We claim that this algorithm takes $O(k(n+m))$ time. First, observe that $G^{\prime}$ has at most $n+m$ edges: for each $i \in\{1, \ldots, \ell\}$, we replace the edges of $E\left(G\left[V_{i}\right]\right)$ by a number of edges equal to $\left|V_{i}\right| \leq\left|E\left(G\left[V_{i}\right]\right)\right|+1$. As we are trying to find an $(X, Y)$-separator of weight at most $k$ in $G^{\prime}$, we can accomplish this by performing at most $k$ rounds of the Ford-Fulkerson max-flow min-cut algorithm, giving the running time $O(k(n+m))$.

Given a set $Z \subseteq\{1, \ldots, \ell\}$, let us define edge set $C_{Z}=\cup_{i \in Z} E\left(G\left[V_{i}\right]\right)$ and vertex set $S_{Z}=\left\{v_{i}: i \in Z\right\}$. The following claim establishes the correctness of the algorithm.

Claim. For any $Z \subseteq\{1, \ldots, \ell\}$,

(i) $r_{M}\left(C_{Z}\right)$ equals the weight of $S_{Z}$;

(ii) $C_{Z}$ is an $(X, Y)$-cut in $G$ iff $S_{Z}$ is an $(X, Y)$-separator in $G^{\prime}$.

To prove (i), note first that the vertex set of each connected component of $G\left[C_{Z}\right]$ is some $V_{i}$. Furthermore, as the two endpoints of each edge in $M$ is in the same $V_{i}$, it is also true in the graph $G\left[C_{Z} \cup M\right]$ that the vertex set of each connected component is some $V_{i}$. Thus, each connected component of $G\left[C_{Z} \cup M\right] / M$ is obtained from a set $V_{i}$ by identifying some vertices. We obtain that $r_{M}\left(C_{Z}\right)=\sum_{i \in Z} r_{M}\left(E\left(G\left[V_{i}\right]\right)\right)$ equals the weight of $S_{Z}$. 
To prove (ii), suppose that $C_{Z}$ is an $(X, Y)$-cut in $G$; we need to show that $S_{Z}$ is an $(X, Y)$-separator in $G^{\prime}$. By way of contradiction, assume that $G^{\prime}$ contains an $(X, Y)$-path $W$ avoiding $S_{Z}$. For each segment of $W$ of the form $x v_{i} y$ with $x, y \in V_{i}, i \notin Z$, we replace it by an $x, y$-path in $G\left[V_{i}\right]$ (recall that the neighbors of $v_{i}$ are in $\left.V_{i}\right)$. We obtain an $(X, Y)$-walk in $G$ avoiding $C_{Z}$, a contradiction.

Conversely, suppose that $S_{Z}$ is an $(X, Y)$-separator in $G^{\prime}$, and let us show that $C_{Z}$ is an $(X, Y)$-cut in $G$. By way of contradiction, assume that $G$ contains an $(X, Y)$-path $W$ avoiding $C_{Z}$. Then $W$ can be partitioned as $W_{1} W_{2} \ldots W_{r}$, where each $W_{j}$ is a maximal subpath of $W$ included in a set $V_{i}$ (possibly, $W_{j}$ contains a single vertex). Each $W_{j}$ that has at least two vertices is an $x, y$-path included in a component $V_{i}$ with $i \notin Z$; we replace it by a path of the form $x v_{i} y$, to obtain an $(X, Y)$-walk in $G^{\prime}$ avoiding $S_{Z}$, a contradiction.

\section{Reduction to the constrained version}

In this section, we describe a randomized reduction mapping an instance $I=$ $(G, k, X, Y, M)$ of RANK-Cut to an instance $I^{\prime}=(G, k, X, Y, M, P)$ of ConSTRAINED RANK-CUT.

The first step of the reduction identifies a set of relevant edges $E_{\text {rel }} \subseteq E(G)$ that spans a graph of bounded degree. It relies on the notion of important separators introduced in [22, which we recall now. Fix two disjoint sets $X, Y \subseteq$ $V(G)$, and let $S$ be an $(X, Y)$-separator in $G$. We denote by $\operatorname{Reach}_{G}(X, S)$ the set of vertices of $G$ reachable from $X$ in $G \backslash S$; note that $\operatorname{Reach}_{G}(X, S)$ is disjoint from $Y$. We say that $S$ is an important $(X, Y)$-separator if (i) $S$ is an inclusion-wise minimal $(X, Y)$-separator, (ii) there is no $(X, Y)$-separator $S^{\prime}$ with $\left|S^{\prime}\right| \leq|S|$ and $\operatorname{Reach}_{G}(X, S) \subset \operatorname{Reach}_{G}\left(X, S^{\prime}\right)$. We have the following result:

Lemma 8 ([22,46]). Let $k$ be a nonnegative integer. There are at most $4^{k}$ important $(X, Y)$-separators of size $\leq k$, and they can be enumerated in time $O\left(4^{k} k(n+m)\right)$.

We now describe the construction of the set $E_{\text {rel }}$. Starting with $G$, we construct a graph $G^{\prime}$ by subdividing each edge $e$ with a vertex $z_{e}$. Given two subsets $X, Y \subseteq V(G)$, we denote by $C_{k}(X, Y)$ the union of the important $(X, Y)$-separators of size at most $k$ in the extended graph $G^{\prime}$. As there are are at most $4^{k}$ such separators by Lemma 8, we have $\left|C_{k}(X, Y)\right| \leq k \cdot 4^{k}$. Given a vertex $u \in V(G)$, we denote by $E(u)$ the set of edges of $G$ incident to $u$. We define the set $E_{\text {rel }} \subseteq E(G)$ as follows: (i) for each $u \in V(G)$, let $E_{\text {rel }}(u)=\left\{e \in E(u): z_{e} \in C_{6 k}(X,\{u\}) \cup C_{6 k}(Y,\{u\})\right\}$; (ii) $E_{\text {rel }}$ consists of those edges $u v \in E(G)$ such that $u v \in E_{\text {rel }}(u) \cap E_{\text {rel }}(v)$. By Lemma 8 . $E_{\text {rel }}$ can be constructed in time $O\left(4^{6 k} k \cdot n(n+m)\right)$, as we need to enumerate important separators for $n$ vertices. Furthermore, the graph $G\left[E_{\text {rel }}\right]$ has maximum degree $d=12 k \cdot 4^{6 k}$, as each set $E_{\text {rel }}(u)$ comes from the union of two sets $C_{6 k}(X,\{u\})$ and $C_{6 k}(Y,\{u\})$, each of which has size at most $6 k \cdot 4^{6 k}$. The interest of the set $E_{\text {rel }}$ is that it contains any minimal solution for $I$. 
Lemma 9. Any minimal solution $C$ of a RANK-CUT instance $I$ is included in $E_{\text {rel }}$.

Proof. We show that for every $e=u v \in C$, we have $e \in E_{\text {rel }}(v)$; this will imply that $e \in E_{\text {rel }}(u)$ by symmetry, and thus $e \in E_{\text {rel }}$. As $C$ is a minimal $(X, Y)$ cut, if we define $U$ to be the set of vertices reachable from $X$ in $G \backslash C$, then $X \subseteq U \subseteq V(G) \backslash Y$ holds and $C$ is the set of edges with exactly one endpoint in $U$. Let $C_{X}$ denote the endpoints of $C$ inside $U$, and let $C_{Y}$ denote the endpoints of $C$ inside $V(G) \backslash U$. We suppose that $v \in C_{Y}$, as the case $v \in C_{X}$ is similar. Let us define the vertex set $S$ of $G^{\prime}$ as $S=\left(C_{Y} \backslash v\right) \cup\left\{z_{e}: e \in C \cap E(v)\right\}$. We make the following observations:

- $S$ is an $(X, v)$-separator in $G^{\prime}$, as each $(X, v)$-path in $G$ either goes through $C_{Y} \backslash v$, or goes through an edge of $C$ incident to $v$ (note also that $S$ is disjoint from $X \cup\{v\})$.

- $u \in \operatorname{Reach}_{G^{\prime}}(X, S):$ as $C$ is a minimal $(X, Y)$-cut, there has to be an $(X, u)$ path in $G$ disjoint from $C$, that is, fully contained in $U$, which means that the corresponding path in $G^{\prime}$ avoids $S$.

$-|S| \leq 6 k$. By Lemma 5 the edges in $C$ span at most $6 k$. Every vertex of $C$ can appear in $C_{Y}$ or can be adjacent to $v$, but not both. Therefore, each vertex spanned by $C$ contributes at most one to $S$ and $|S| \leq 6 k$ follows.

By the definition of important separators, there exists an important $(X, v)$ separator $S^{\prime}$ in $G^{\prime}$ such that $\operatorname{Reach}_{G^{\prime}}(X, S) \subseteq \operatorname{Reach}_{G^{\prime}}\left(X, S^{\prime}\right)$ and $\left|S^{\prime}\right| \leq|S|$. As $z_{e}$ is adjacent to $u$ and $v$, as $u \in \operatorname{Reach}_{G^{\prime}}\left(X, S^{\prime}\right)$ and as $S^{\prime}$ is an $(X, v)$ separator in $G^{\prime}$, it follows that $z_{e} \in S^{\prime}$. Now, $S^{\prime} \subseteq C_{6 k}(X,\{v\})$ implies that $z_{e} \in C_{6 k}(X,\{v\})$, and we conclude that $e \in E_{\text {rel }}(v)$.

We construct an instance $I^{\prime}$ of CONSTRAINED RANK-CUT from the instance $I$ of RANK-CuT, by the following random process. Let $p=\frac{1}{6 k d}=2^{-O(k)}$. We color edges of $E_{\text {rel }} \backslash M$ with color black with probability $p$, and with color red otherwise. Let $E_{b}$ denote the set containing the edges of $E_{\text {rel }}$ colored black, as well as the edges of $M$. Consider the subgraph $G_{b}$ of $G$ containing only the edges in $E_{b}$ and let partition $P=\left(V_{1}, \ldots, V_{\ell^{\prime}}\right)$ represent the way the connected components of this subgraph partition $V(G)$ (note that $P$ can have classes that contain only a single vertex). By definition, $G\left[V_{i}\right]$ is connected for every $i$ and the two endpoints of each edge in $M$ is in the same $V_{i}$. Therefore, the Constrained RANK-CUT instance $I^{\prime}=(G, k, X, Y, M, P)$ is a valid instance, as it satisfies both (i) and (ii).

Lemma 10. The following two statements hold:

1. If $I$ is a no-instance of RANK-CUT, then $I^{\prime}$ is a no-instance of CONSTRAINED RANK-CUT.

2. If $I$ is a yes-instance of RANK-CuT, then $I^{\prime}$ is a yes-instance of CONSTRAINED RANK-CuT with probability $2^{-O\left(k^{2}\right)}$. 
Proof. Clearly, if $I^{\prime}$ has a solution $Z$, then $C_{Z}$ is a solution for instance $I$ of RANK-CuT. Conversely, suppose that $I$ has a minimal solution $C \subseteq E(G)$ with $r_{M}(C) \leq k$. Let $U_{1}, \ldots, U_{\ell^{\prime}}$ denote the vertex sets of the connected components of $G[C \cup M]$ (note that this is not necessarily a partition of $V(G)$, as it is possible to have vertices that are not incident to any edge of $C \cup M)$. Let $F$ be a spanning forest of $G[C \cup M]$ containing as many edges of $M$ as possible. Let $B=F \backslash M$; as all these edges are in $C$, we have $B \subseteq E_{\text {rel }}$ by Lemma 9 , and since we have $r_{M}(C) \leq k$ it follows that $|B| \leq k$. Let $R=\cup_{i=1}^{\ell^{\prime}} E_{\text {rel }}\left(U_{i}\right)$, where $E_{\text {rel }}\left(U_{i}\right)$ denotes the set of edges in $E_{\text {rel }}$ with exactly one endpoint in $U_{i}$. By Lemma 5, $C \cup M$ spans at most $6 k$ vertices, thus $\sum_{i=1}^{\ell^{\prime}}\left|U_{i}\right| \leq 6 k$. As each vertex of $V(G)$ has at most $d$ incident edges in $E_{\text {rel }}$, we have $|R| \leq d \sum_{i=1}^{\ell^{\prime}}\left|U_{i}\right| \leq 6 k d=2^{O(k)}$. Now, (i) with probability at least $p^{k}=2^{-O\left(k^{2}\right)}$, every edge of $B$ is colored black, (ii) with probability at least $\left(1-\frac{1}{6 k d}\right)^{6 k d} \geq \frac{1}{4}$, every edge of $R$ is colored red (indeed, the function $x \mapsto\left(1-\frac{1}{x}\right)^{x}$ is increasing on $\left[1,+\infty\right.$ [ and is thus $\geq \frac{1}{4}$ for $x \geq 2$ ). These two events are independent, as they involve disjoint sets of edges. Suppose that both events happen. Then, $E_{b}$ contains all edges of $F$, but no edge of $R$. Consider the subgraph $G_{b}$ of $G$ containing only the edges in $E_{b}$ and let partition $P=\left(V_{1}, \ldots, V_{\ell}\right)$ represent the way the connected components of this subgraph partition $V(G)$. Then every $U_{i}$ is one class of this partition. Thus $C^{\prime}=\cup_{i=1}^{\ell^{\prime}} E\left(G\left[U_{i}\right]\right)$ is a solution for instance $I^{\prime}$ (as $C^{\prime} \supseteq C \cup M$ and $\left.r_{M}\left(C^{\prime}\right)=r_{M}(C) \leq k\right)$. We conclude that $I^{\prime}$ is a yes-instance with probability $2^{-O\left(k^{2}\right)}$.

From Lemmas 7 and 10, we obtain:

Theorem 11. RANK-CUT has a randomized FPT algorithm with running time $2^{O\left(k^{2}\right)} n m$.

Proof. Let $I=(G, k, X, Y, M)$ be an instance of RAnK-Cut. We first remove all isolated vertices of $G$ in time $O(n+m)$, obtaining a graph $G$ for which each connected component has at least two vertices, ensuring that $n+m=O(m)$. We then compute the set $E_{\text {rel }}$ in time $O\left(4^{6 k} \mathrm{knm}\right)$, and we construct the instance $I^{\prime}$ of Constrained RAnK-Cut by random selection as described above. This instance $I^{\prime}$ can be solved in time $O(k(n+m))$ by Lemma 7. By Lemma 10, the probability of a correct answer is at least $p_{\text {correct }}=2^{-O\left(k^{2}\right)}$. Thus repeating this process $\left\lceil\frac{1}{p_{\text {correct }}}\right\rceil=2^{O\left(k^{2}\right)}$ times yields a randomized FPT algorithm for RANK-CUT running in time $2^{O\left(k^{2}\right)} n m$ and having success probability $\left(1-p_{\text {correct }}\right)^{\frac{1}{p_{\text {correct }}}} \geq \frac{1}{4}$.

\section{Derandomization}

We now derandomize the proofs of Lemma 10 and Theorem 11 using the standard technique of splitters. Given integers $n, s, t$, an $(n, s, t)$-splitter is a family $\mathcal{F}$ of functions $f:[n] \rightarrow[t]$ such that for every $S \subseteq[n]$ with $|S|=s$, there is a function of $\mathcal{F}$ that is injective of $S$. Naor et al. [26] give a deterministic construction of an 
$\left(n, s, s^{2}\right)$-splitter of size $O\left(s^{6} \log s \log n\right)$. We can use this splitter construction to build a family of colorings of $E_{\text {rel }}$ to replace the randomized selection of colors in Lemma 10. The proof of Lemma 10 analyzed the probability of the event that a certain set $B$ of edges is colored black and at the same time a certain set $R$ of edges is colored red. By setting the parameters of splitters appropriately, we can ensure that at least one coloring in the family has this property. The $\left(n, s, s^{2}\right)$-splitter of Naor et al. 26] can be constructed in polynomial time, but unfortunately the exact running time is not stated. Therefore, in the following theorem, we do not specify the polynomial factors of the running time.

Theorem 12. RANK-CUT has a deterministic FPT algorithm with running time $2^{O\left(k^{2}\right)} n^{O(1)}$.

Proof. Consider an instance $I=(G, k, X, Y, M)$ of RAnK-CuT. We first construct the set $E_{\text {rel }}$ as in Section 5, and we identify $E_{\text {rel }} \backslash M$ with the set $\left[\mathrm{m}^{\prime}\right]$ where $m^{\prime}=\left|E_{\text {rel }} \backslash M\right|$. Let $s=k+4 k d=2^{O(k)}$. Using the result of [26], we construct an $\left(m^{\prime}, s, s^{2}\right)$-splitter $\mathcal{F}$ of size $O\left(s^{6} \log s \log m\right)$. Instead of randomly coloring the elements of $E_{\text {rel }} \backslash M$, we go through the following deterministic family of colorings: for every $f \in \mathcal{F}$ and every subset $U \subseteq\left[s^{2}\right]$ of size at most $k$, we color $e \in E_{\text {rel }} \backslash M$ black if and only if $f(e) \in U$. For each such coloring, we perform the reduction to Constrained RANK-CuT as in Lemma 10] and then solve the instance using the algorithm of Lemma 7. We return "yes" if and only if at least one of the resulting Constrained RAnK-Cut instances is a yes-instance.

It is clear that if one of the CONSTRAInED RANK-CUT instances is a yesinstance, then $I$ is a yes-instance of RANK-CUT. Conversely, suppose that $I$ is a yes-instance and let $B$ and $R$ be the set of edges defined in the proof of Lemma 10. As $|B|+|R| \leq s$, there is a function $f \in \mathcal{F}$ that is injective on $B \cup R$ and there is a set $U \subseteq\left[s^{2}\right]$ of size at most $k$ such that $b \in B \cup R$ satisfies $b \in B$ if and only $f(b) \in U$. For this choice of $f$ and $U$, the algorithm considers a coloring that colors $B$ black and $R$ red. Therefore, the reduction creates a yes-instance of Constrained RAnK-Cut.

Theorems 11 and 12 respectively give randomized and deterministic FPT algorithms for RANK-CuT. Combining them with Lemmas 3 and [6, we obtain (i) a $2^{O\left(k^{2}\right)} \mathrm{nm}$ randomized algorithm for Bipartite Contraction, (ii) a $2^{O\left(k^{2}\right)} n^{O(1)}$ deterministic algorithm BipARTite Contraction. This establishes Theorem 1 stated in the introduction.

\section{Concluding remarks}

We have obtained a randomized $2^{O\left(k^{2}\right)} \mathrm{nm}$ algorithm for BIPARTITE CONTRACTION. Can the dependence on $k$ be improved? It seems plausible that the problem admits a $2^{O(k)} n^{O(1)} \mathrm{FPT}$ algorithm, as such algorithms are known for EDGE BIPARTIZATION [11] as well as for other edge contraction problems [12. We note 
that important separators are a common feature of [13] and of our algorithm, so they could be the key to further improvements.

Regarding kernelization, Heggernes et al. [13] asked whether BipARTITE CONTRACTION has a polynomial kernel. While this question is still open, it is now known that OdD Cycle Transversal (and thus Edge Bipartization) have randomized polynomial kernels [18. As EDGE BipARTIZATION reduces to BIPARTITE CONTRACTION, this raises the question whether the matroid-based techniques of [18,19] can be applied to the more general BipARTITE ConTraCTION as well. The notion of rank in the RANK-CUT problem is the same as the notion of rank in graphic matroids, hence it is possible that the rank constraint can be incorporated into the arguments of [1819. based on linear representation of matroids.

\section{References}

1. N. Alon, R. Yuster, and U. Zwick. Color-coding. J. ACM, 42(4):844-856, 1995.

2. Y. Cao and D. Marx. Interval Deletion is Fixed-Parameter Tractable. CoRR, abs/1211.5933, 2012.

3. J. Chen, F. V. Fomin, Y. Liu, S. Lu, and Y. Villanger. Improved algorithms for feedback vertex set problems. J. Comput. Syst. Sci., 74(7):1188-1198, 2008.

4. J. Chen, Y. Liu, and S. Lu. An Improved Parameterized Algorithm for the Minimum Node Multiway Cut Problem. Algorithmica, 55(1):1-13, 2009.

5. J. Chen, Y. Liu, S. Lu, B. O'Sullivan, and I. Razgon. A fixed-parameter algorithm for the directed feedback vertex set problem. J. ACM, 55(5), 2008.

6. R. H. Chitnis, M. Hajiaghayi, and D. Marx. Fixed-Parameter Tractability of Directed Multiway Cut Parameterized by the Size of the Cutset. To appear in SIAM Journal on Computing. http://arxiv.org/abs/1110.0259.

7. M. Cygan, M. Pilipczuk, and M. Pilipczuk. On Group Feedback Vertex Set Parameterized by the Size of the Cutset. In Proceedings $W G$ 2012, volume 7551 of LNCS, pages 194-205, 2012.

8. F. K. Dehne, M. R. Fellows, M. A. Langston, F. A. Rosamond, and K. Stevens. An $O\left(2^{O(k)} n^{3}\right)$ FPT algorithm for the undirected feedback vertex set problem. Theor. Comput. Syst., 41(3):479-492, 2007.

9. P. A. Golovach, P. van't Hof, and D. Paulusma. Obtaining Planarity by Contracting Few Edges. In Proceedings MFCS 2012, volume 7464 of LNCS, pages 455-466, 2012.

10. S. Guillemot. FPT algorithms for path-transversals and cycle-transversals problems. Discrete Optimization, 8(1):61-71, 2011.

11. J. Guo, J. Gramm, F. Hüffner, R. Niedermeier, and S. Wernicke. Compressionbased fixed-parameter algorithms for feedback vertex set and edge bipartization. J. Comput. Syst. Sci., 72(8):1386-1396, 2006.

12. P. Heggernes, P. van't Hof, B. Lévêque, D. Lokshtanov, and C. Paul. Contracting Graphs to Paths and Trees. In Proceedings IPEC 2011, volume 7112 of LNCS, pages 55-66, 2011.

13. P. Heggernes, P. van't Hof, D. Lokshtanov, and C. Paul. Obtaining a Bipartite Graph by Contracting Few Edges. In Proceedings FSTTCS 2011, volume 13 of LIPIcs, pages 217-228, 2011.

14. K. ichi Kawarabayashi. Planarity Allowing Few Error Vertices in Linear Time. In FOCS, pages 639-648, 2009. 
15. Y. Iwata, K. Oka, and Y. Yoshida. Linear-Time FPT Algorithms via Network Flow. CoRR, abs/1307.4927, 2013.

16. H. Kaplan, R. Shamir, and R. E. Tarjan. Tractability of parameterized completion problems on chordal, strongly chordal, and proper interval graphs. SIAM J. Comput., 28(5):1906-1922, 1999.

17. K. Kawarabayashi and B. A. Reed. An (almost) Linear Time Algorithm for Odd Cycle Transversal. In Proceedings SODA 2010, pages 365-378, 2010.

18. S. Kratsch and M. Wahlström. Compression via Matroids: a Randomized Polynomial Kernel for Odd Cycle Transversal. In Proceedings SODA 2012, pages 94-103, 2012.

19. S. Kratsch and M. Wahlström. Representative sets and irrelevant vertices: New tools for kernelization. In Proceedings FOCS 2012, 2012. To appear.

20. D. Lokshtanov, N. S. Narayanaswamy, V. Raman, M. S. Ramanujan, and S. Saurabh. Faster Parameterized Algorithms using Linear Programming. CoRR, abs/1203.0833, 2012.

21. D. Lokshtanov, S. Saurabh, and S. Sikdar. Simpler Parameterized Algorithm for OCT. In Proceedings IWOCA 2009, volume 5874 of LNCS, pages 380-384, 2009.

22. D. Marx. Parameterized graph separation problems. Theoretical Computer Science, 351(3):394-406, 2006.

23. D. Marx. Chordal deletion is fixed-parameter tractable. Algorithmica, 57(4):747$768,2010$.

24. D. Marx, B. O'Sullivan, and I. Razgon. Finding small separators in linear time via treewidth reduction. ACM Transactions on Algorithms, 2012. To appear.

25. D. Marx and I. Schlotter. Obtaining a planar graph by vertex deletion. Algorithmica, 62(3-4):807-822, 2012.

26. M. Naor, L. J. Schulman, and A. Srinivasan. Splitters and near-optimal derandomization. In Proceedings FOCS 1995, pages 182-191, 1995.

27. M. S. Ramanujan and S. Saurabh. Linear Time Parameterized Algorithms via Skew-Symmetric Multicuts. CoRR, abs/1304.7505, 2013.

28. B. A. Reed, K. Smith, and A. Vetta. Finding odd cycle transversals. Oper. Res. Lett., 32(4):299-301, 2004.

29. R. van Bevern, C. Komusiewicz, H. Moser, and R. Niedermeier. Measuring Indifference: Unit Interval Vertex Deletion. In $W G$, pages 232-243, 2010.

30. P. van 't Hof and Y. Villanger. Proper Interval Vertex Deletion. Algorithmica, 65(4):845-867, 2013.

31. Y. Villanger, P. Heggernes, C. Paul, and J. A. Telle. Interval Completion Is Fixed Parameter Tractable. SIAM J. Comput., 38(5):2007-2020, 2009. 